\title{
Weak amenability of Banach algebras with respect to characters
}

\author{
Rasoul Nasr-Isfahani Somayeh Shahmoradi \\ Sima Soltani Renani
}

\begin{abstract}
For a Banach algebra $\mathcal{A}$, we introduce and investigate weak amenability of $\mathcal{A}$ with respect to a character. We give some necessary conditions for the weak amenability of $\mathcal{A}$ with respect to a character and describe a class of Banach algebras that are not weakly amenable with respect to characters. Finally, we give examples of Banach algebras which are weakly amenable with respect to characters but neither weakly amenable nor amenable with respect to characters.
\end{abstract}

\section{Introduction}

An important result by Johnson [10] is that a locally compact group $G$ is amenable if and only if the group algebra $L^{1}(G)$ is amenable as a Banach algebra; that is, the first cohomology groups $H^{1}\left(L^{1}(G), \mathcal{X}^{*}\right)$ vanishes for all Banach $L^{1}(G)$-bimodules $\mathcal{X}$. This equivalence does not remain true for the convolution semigroup algebra $\ell^{1}(S)$ of a discrete semigroup $S$.

Lau [16] introduced and investigated a weaker property on $F$-algebras, called left amenability, by relativizing amenability to the identity element of the dual $W^{*}$-algebra. Recall that an F-algebras is a Banach algebra $\mathcal{L}$ which is the unique predual of a $W^{*}$-algebra $M$ and the identity element $u$ of $M$ is a character on $\mathcal{L}$; later, in Pier [27], F-algebras were called Lau algebras. In fact, the Lau algebra $\mathcal{L}$

Received by the editors in October 2015 - In revised form in June 2016.

Communicated by F. Bastin.

2010 Mathematics Subject Classification : Primary 46H05, 46H25, 47B47. Secondary 43A10.

Key words and phrases : Banach algebra, Banach module, character, derivation, dual space, weakly amenable. 
is said to be left amenable if $H^{1}\left(\mathcal{L} ; \mathcal{X}^{*}\right)=\{0\}$ for all Banach $\mathcal{L}$-bimodules $\mathcal{X}$ with the left action defined by $l \cdot x=u(l) x$ for all $l \in \mathcal{L}$ and $x \in \mathcal{X}$; see also [6], [14], [15], [17], [19], [20] and [21]. Lau [16] proved that $\ell^{1}(S)$ is left amenable if and only if $S$ is left amenable.

The class of Lau algebras includes the Fourier algebra $A(G)$ and the group algebra $L^{1}(G)$ of a locally compact group $G$, as well as the measure algebra $M(S)$ of a locally compact topological semigroup $S$; see Lau [16]. It also includes the Fourier-Stieltjes algebra $B(G)$ of any topological group $G$; see Lau and Ludwig [19].

A considerable generalization of the concept of left amenability was introduced by Kaniuth, Lau and Pym [12] (see also Monfared [22]) for a Banach algebra $\mathcal{A}$ with respect to an arbitrary character $\phi$ on $\mathcal{A}$; in fact, the Banach algebra $\mathcal{A}$ is called $\phi$-amenable if $H^{1}\left(\mathcal{A} ; \mathcal{X}^{*}\right)$ vanishes for all Banach $\mathcal{A}$-bimodules $\mathcal{X}$ for which the left module action of $\mathcal{A}$ on $\mathcal{X}$ is defined by $a \cdot x=\phi(a) x$ for $a \in \mathcal{A}$ and $x \in \mathcal{X}$. For some related works on amenability of Banach algebras with respect to a character, see $[1,2,5,13,23,24,25,26]$.

Moreover, the notion of weak amenability for an arbitrary Banach algebra was introduced and studied by Johnson [11]; the Banach algebra $\mathcal{A}$ is called weakly amenable if $H^{1}\left(\mathcal{A}, \mathcal{A}^{*}\right)=\{0\}$, where the dual space $\mathcal{A}^{*}$ of $\mathcal{A}$ is equipped with the Arens module structure; see also the recent works $[7,18]$.

Our goal in this work is to introduce and to study weak character amenability of a Banach algebra $\mathcal{A}$ as a new notion of amenability. In Section 2, we show that if $\mathcal{A}$ has a bounded left approximate identity, then $\mathcal{A}$ is weakly character amenable; however the converse is not true. In Section 3, we will focus on a special class of module extension Banach algebras to characterize their derivations; we also use this result to give a class of Banach algebras that are not weakly character amenable. We finally compare the notion of weak character amenability with some different versions of amenability.

\section{Weak character amenability}

Let $\mathcal{A}$ be a Banach algebra and let $\mathcal{X}$ be a Banach $\mathcal{A}$-bimodule. Then the dual $\mathcal{X}^{*}$ of $\mathcal{X}$, has an Arens $\mathcal{A}$-bimodule structure defined by

$$
\begin{aligned}
& (a \cdot \Lambda)(x)=\Lambda(x \cdot a) \\
& (\Lambda \cdot a)(x)=\Lambda(a \cdot x)
\end{aligned}
$$

for all $a \in \mathcal{A}, x \in \mathcal{X}$ and $\Lambda \in \mathcal{X}^{*}$.

A derivation of $\mathcal{A}$ into $\mathcal{X}^{*}$ is a linear mapping $D: \mathcal{A} \longrightarrow \mathcal{X}^{*}$ such that

$$
D(a b)=D(a) \cdot b+a \cdot D(b)
$$

for all $a, b \in \mathcal{A}$. For each $\Lambda \in \mathcal{X}^{*}$, the mapping $\operatorname{ad}_{\Lambda}: \mathcal{A} \rightarrow \mathcal{X}^{*}$ defined by

$$
\operatorname{ad}_{\Lambda}(a)=a \cdot \Lambda-\Lambda \cdot a
$$

is a derivation, called the inner derivation associated with $\Lambda$. 
Let $\mathcal{Y}$ be a Banach right $\mathcal{A}$-module and $\phi \in \Delta(\mathcal{A}) \cup\{0\}$, where $\Delta(\mathcal{A})$ is the spectrum of $\mathcal{A}$ consisting of all characters from $\mathcal{A}$ into the complex numbers. We denote by ${ }_{\phi} \mathcal{Y}$ the Banach $\mathcal{A}$-bimodule $\mathcal{Y}$ such that the left action satisfies

$$
a \cdot y=\phi(a) y
$$

for all $a \in \mathcal{A}$ and $y \in \mathcal{Y}$. Then $\left.{ }_{\phi} \mathcal{Y}\right)^{*}$ is also a Banach $\mathcal{A}$-bimodule with the natural dual actions.

We always consider $\mathcal{A}$ as a Banach right $\mathcal{A}$-module with the actions obtained from the multiplication of $\mathcal{A}$. Then ${ }_{\phi} \mathcal{A}$ is a Banach $\mathcal{A}$-bimodule with the left and right actions

$$
a \cdot x=\phi(a) x \quad \text { and } \quad x \cdot a=x a
$$

for all $a, x \in \mathcal{A}$, respectively. Moreover, $\left({ }_{\phi} \mathcal{A}\right)^{*}$ is a Banach $\mathcal{A}$-bimodule with the left and right actions defined by

$$
a \cdot f=a f \quad \text { and } \quad f \cdot a=\phi(a) f
$$

for all $a \in \mathcal{A}$ and $f \in \mathcal{A}^{*}$, respectively; here $a f \in \mathcal{A}^{*}$ is defined by $(a f)(b)=f(b a)$ for all $b \in \mathcal{A}$.

Definition 2.1. Let $\mathcal{A}$ be a Banach algebra and let $\phi \in \Delta(\mathcal{A}) \cup\{0\}$. We say that $\mathcal{A}$ is weakly $\phi$-amenable if

$$
H^{1}\left(\mathcal{A},\left({ }_{\phi} \mathcal{A}\right)^{*}\right)=\{0\} .
$$

We also say that $\mathcal{A}$ is weakly character amenable whenever $\mathcal{A}$ is weakly $\phi$-amenable for all $\phi \in \Delta(\mathcal{A}) \cup\{0\}$.

In the following, we introduce a large class of weakly character amenable Banach algebras.

Theorem 2.2. Let $\mathcal{A}$ be a Banach algebra. If $\mathcal{A}$ has a bounded left approximate identity, then $\mathcal{A}$ is weakly character amenable. In particular, the unitization $\mathcal{A}^{\sharp}$ of $\mathcal{A}$ is weakly character amenable.

Proof. For $\phi \in \Delta(\mathcal{A}) \cup\{0\}$, suppose that $D: \mathcal{A} \rightarrow\left({ }_{\phi} \mathcal{A}\right)^{*}$ is a derivation and let $\left(e_{\gamma}\right)_{\gamma \in \Gamma}$ be a bounded left approximate identity for $\mathcal{A}$. For $\gamma \in \Gamma$, define the functional $f_{\gamma} \in \mathcal{A}^{*}$ by

$$
f_{\gamma}(a):=\left\langle D(a), e_{\gamma}\right\rangle
$$

for all $a \in \mathcal{A}$. The bounded net $\left(f_{\gamma}\right)_{\gamma \in \Gamma} \subseteq \mathcal{A}^{*}$ has a subnet $\left(f_{\beta}\right)_{\beta \in \Gamma}$ converging to a point $f$ in the weak*-topology of $\mathcal{A}^{*}$. Then for each $a, b \in A$,

$$
\begin{aligned}
\left\langle\operatorname{ad}_{f}(a), b\right\rangle & =(a f)(b)-\phi(a) f(b) \\
& =f(b a)-\phi(a) f(b) \\
& =\lim _{\beta} f_{\beta}(b a)-\phi(a) f_{\beta}(b) \\
& =\lim _{\beta}\left\langle D(b a), e_{\beta}\right\rangle-\phi(a)\left\langle D(b), e_{\beta}\right\rangle \\
& =\lim _{\beta}\left\langle D(a), e_{\beta} b\right\rangle \\
& =\langle D(a), b\rangle .
\end{aligned}
$$

This means that $D=\operatorname{ad}_{f}$ which completes the proof. 
The following example shows that Theorem 2.2 does not remain valid for all Banach algebras with approximate identity (not necessarily bounded).

Example 2.3. Let $\mathcal{A}$ be a Banach algebra without right identity such that $\mathcal{A}$ is isometrically isomorphic to the dual space $\mathcal{A}^{*}$. Then $\mathcal{A}$ is not weakly 0 -amenable; indeed, the identity map

$$
\mathrm{I}: \mathcal{A} \longrightarrow\left({ }_{0} \mathcal{A}\right)^{*}
$$

is a derivation which is not inner. In particular, the discrete Banach algebra $\ell^{2}(\mathbb{N})$ of all sequences of complex numbers $a:=(a(n))$ with

$$
\|a\|_{2}:=\left(\sum_{n=1}^{\infty}|a(n)|^{2}\right)^{\frac{1}{2}}<\infty
$$

endowed with pointwise product is not weakly 0 -amenable. Note that $\ell^{2}(\mathbb{N})$ has a left approximate identity.

In the sequel, we investigate the relationship between weak $\phi$-amenability of $\mathcal{A}$ and the 2-nd Hochschild cohomology group of $\mathcal{A}$ with coefficients in $\mathbb{C}$. We first state some preliminary results and notations.

Let $\mathcal{A}$ be a Banach algebra and let $\mathcal{X}$ be a Banach $\mathcal{A}$-bimodule. Then the space

$$
L^{k}(\mathcal{A}, \mathcal{X}):=\left\{T: \prod_{n=1}^{k} \mathcal{A} \rightarrow \mathcal{X}: \mathrm{T} \text { is bounded and } k \text {-linear }\right\}
$$

becomes a Banach $\mathcal{A}$-bimodule, through

$$
(a \cdot T)\left(a_{1}, \ldots, a_{k}\right):=a \cdot T\left(a_{1}, \ldots, a_{k}\right)
$$

and

$$
\begin{aligned}
(T \cdot a)\left(a_{1}, \ldots, a_{k}\right): & =T\left(a a_{1}, \ldots, a_{k}\right) \\
& +\sum_{j=1}^{k-1}(-1)^{j} T\left(a, a_{1}, \ldots, a_{j} a_{j+1}, \ldots, a_{k}\right) \\
& +(-1)^{k} T\left(a, a_{1}, \ldots, a_{k-1}\right) \cdot a_{k} .
\end{aligned}
$$

for all $T \in L^{k}(\mathcal{A}, \mathcal{X})$ and $a, a_{1}, \ldots, a_{k} \in \mathcal{A}$; for more details see [28]. Now, consider the operators

$$
\delta^{1}: L^{1}(\mathcal{A}, \mathcal{X}) \rightarrow L^{2}(\mathcal{A}, \mathcal{X})
$$

and

$$
\delta^{2}: L^{2}(\mathcal{A}, \mathcal{X}) \rightarrow L^{3}(\mathcal{A}, \mathcal{X})
$$

with formulates

$$
\begin{gathered}
\left(\delta^{1} S\right)\left(a_{1}, a_{2}\right):=a_{1} \cdot S a_{2}-S\left(a_{1} a_{2}\right)+S\left(a_{1}\right) \cdot a_{2} \\
\left(\delta^{2} T\right)\left(a_{1}, a_{2}, a_{3}\right):=a_{1} \cdot T\left(a_{2}, a_{3}\right)-T\left(a_{1} a_{2}, a_{3}\right)+T\left(a_{1}, a_{2} a_{3}\right)-T\left(a_{1}, a_{2}\right) \cdot a_{3}
\end{gathered}
$$


for all $T \in L^{2}(\mathcal{A}, \mathcal{X}), S \in L^{1}(\mathcal{A}, \mathcal{X})$ and $a_{1}, a_{2}, a_{3} \in \mathcal{A}$. Then the 2-th Hochschild cohomology group of $\mathcal{A}$ with coefficients in $\mathcal{X}$ is defined by

$$
H^{2}(\mathcal{A}, \mathcal{X}):=Z^{2}(\mathcal{A}, \mathcal{X}) / N^{2}(\mathcal{A}, \mathcal{X})
$$

where

$$
N^{2}(\mathcal{A}, \mathcal{X}):=\operatorname{ran}\left(\delta^{1}\right)
$$

and

$$
Z^{2}(\mathcal{A}, \mathcal{X}):=\operatorname{ker}\left(\delta^{2}\right)
$$

Let $\mathbb{C}$ be the Banach space of complex numbers. We denote by ${ }_{0} \mathbb{C}_{\phi}$ the Banach $\mathcal{A}$-bimodule endowed with the actions defined by

$$
a \cdot \lambda=0 \quad \text { and } \quad \lambda \cdot a=\phi(a) \lambda
$$

for all $a \in \mathcal{A}, \lambda \in \mathbb{C}$ and $\phi \in \Delta(\mathcal{A}) \cup\{0\}$. In Theorem 2.2, we show that $A$ is weakly $\phi$-amenable for all Banach algebras $\mathcal{A}$ with a bounded left approximate identity. In the following result, we extend that result to a considerably larger family of Banach algebras; that is, Banach algebras $\mathcal{A}$ for which $H^{2}\left(\mathcal{A}_{, 0} \mathbb{C}_{\phi}\right)=\{0\}$ for all $\phi \in \Delta(\mathcal{A}) \cup\{0\}$.

Theorem 2.4. Let $\mathcal{A}$ be a Banach algebra and let $\phi \in \Delta(\mathcal{A}) \cup\{0\}$. Consider the following statements

(a) $\mathcal{A}$ has a bounded left approximate identity.

(b) $H^{2}\left(\mathcal{A},{ }_{0} \mathbb{C}_{\phi}\right)=\{0\}$.

(c) $\mathcal{A}$ is weakly $\phi$-amenable.

Then we have $(a) \Longrightarrow(b) \Longrightarrow(c)$.

Proof. That (a) implies (b) follows from the fact that $H^{2}\left(\mathcal{A}, \mathcal{X}^{*}\right)=\{0\}$ for all Banach $\mathcal{A}$-bimodules $\mathcal{X}$ with the right module action $x \cdot a=0(a \in \mathcal{A}, x \in \mathcal{X})$; see [10], Proposition 1.5.

To show that (b) implies (c), consider the short exact sequence

$$
\sum: 0 \rightarrow{ }_{\phi} \mathcal{A} \stackrel{i}{\rightarrow} \phi \mathcal{A}^{\sharp} \stackrel{\phi_{\infty}}{\rightarrow}\left({ }_{0} \mathbb{C}_{\phi}\right)^{*} \rightarrow 0
$$

of Banach $\mathcal{A}$-bimodules, where $i$ is the natural embedding and $\phi_{\infty}(a, \lambda)=\lambda$ for all $a \in \mathcal{A}$ and $\lambda \in \mathbb{C}$. The sequence $\sum$ is admissible; that is, $\phi_{\infty}$ has a bounded right inverse. Hence its dual $\sum^{*}$ is admissible; see [3], Theorem 2.8.31. We therefore have a long exact sequence which contains the subsequence

$$
\cdots \rightarrow H^{1}\left(\mathcal{A},\left({ }_{\phi} \mathcal{A}^{\sharp}\right)^{*}\right) \rightarrow H^{1}\left(\mathcal{A},\left({ }_{\phi} \mathcal{A}\right)^{*}\right) \rightarrow H^{2}\left(\mathcal{A},{ }_{0} \mathbb{C}_{\phi}\right) \rightarrow \ldots
$$

by Theorem 2.8 .25 of [3]. Let $\phi^{\sharp} \in \Delta\left(\mathcal{A}^{\sharp}\right)$ denote the character defined by

$$
\phi^{\sharp}(a, \lambda)=\phi(a)+\lambda
$$

for all $a \in \mathcal{A}$ and $\lambda \in \mathbb{C}$. Suppose that $D: \mathcal{A} \longrightarrow\left({ }_{\phi} \mathcal{A}^{\sharp}\right)^{*}$ is a derivation and extend derivation $D$ to a derivation $D^{\sharp}: \mathcal{A}^{\sharp} \longrightarrow\left({ }_{\phi^{\sharp}} \mathcal{A}^{\sharp}\right)^{*}$ given by

$$
D^{\sharp}(a, \lambda):=D(a)
$$


for all $a \in \mathcal{A}$ and $\lambda \in \mathbb{C}$. Since $\mathcal{A}^{\sharp}$ is weakly character amenable, $D$ is inner and

$$
H^{1}\left(\mathcal{A},\left(\phi \mathcal{A}^{\sharp}\right)^{*}\right)=\{0\} .
$$

But $H^{2}\left(\mathcal{A},{ }_{0} \mathbb{C}_{\phi}\right)=\{0\}$ and so

$$
H^{1}\left(\mathcal{A},\left({ }_{\phi} \mathcal{A}\right)^{*}\right)=\{0\} ;
$$

that is, $\mathcal{A}$ is weakly $\phi$-amenable.

Let $\Omega$ be a set and let $\ell^{1}(\Omega)=\left\{a: \Omega \longrightarrow \mathbb{C}\left|\|a\|_{1}:=\sum_{s \in \Omega}\right| a(s) \mid<\infty\right\}$. Then $\ell^{1}(\Omega)$ with respect to the pointwise operations and the norm $\|.\|_{1}$ is a Banach algebra. We have

$$
\Delta\left(\ell^{1}(\Omega)\right)=\left\{\phi_{s}: s \in \Omega\right\},
$$

where $\phi_{s} \in \ell^{1}(\Omega)^{*}$ is the functional defined by

$$
\phi_{s}(a)=a(s)
$$

for all $a \in \ell^{1}(\Omega)$. Let us recall that $\ell^{1}(\Omega)$ has no bounded left approximate identity if $\Omega$ is infinite.

We continue this section by the following result which shows that the statements (a) and (b) in Theorem 2.4 are not equivalent.

Proposition 2.5. Let $\Omega$ be a set. Then $H^{2}\left(\ell^{1}(\Omega),{ }_{0} \mathbb{C}_{\phi}\right)=\{0\}$ for all $\phi \in \Delta\left(\ell^{1}(\Omega)\right) \cup$ $\{0\}$.

Proof. Fix $s \in \Omega$ and consider $\phi_{s} \in \Delta\left(\ell^{1}(\Omega)\right)$. We know that

$$
H^{2}\left(\ell^{1}(\Omega),{ }_{0} \mathbb{C}_{\phi_{s}}\right)=H^{1}\left(\ell^{1}(\Omega), L^{1}\left(\ell^{1}(\Omega),{ }_{0} \mathbb{C}_{\phi_{s}}\right)\right)
$$

by Theorem 2.4.6 of [28]. Also, we have

$$
H^{1}\left(\ell^{1}(\Omega), L^{1}\left(\ell^{1}(\Omega),{ }_{0} \mathbb{C}_{\phi_{s}}\right)\right)=H^{1}\left(\ell^{1}(\Omega), \ell^{1}(\Omega)^{*}\right),
$$

where $\ell^{1}(\Omega)^{*}$ is a Banach $\ell^{1}(\Omega)$-bimodule with the following module actions

$$
a \cdot f=0, \quad f \cdot a=a f-f(a) \phi_{s}
$$

for all $a \in \ell^{1}(\Omega)$ and $f \in \ell^{1}(\Omega)^{*}$. Therefore, it is sufficient to prove that

$$
H^{1}\left(\ell^{1}(\Omega), \ell^{1}(\Omega)^{*}\right)=\{0\} .
$$

For this end, let $D: \ell^{1}(\Omega) \rightarrow \ell^{1}(\Omega)^{*}$ be a derivation and define

$$
g(t):=-\left\langle D\left(e_{t}\right), e_{t}\right\rangle
$$

for $t \in \Omega$, where $e_{t}$ is defined on $\Omega$ by $e_{t}(u)=1$ for $u=t$ and $e_{t}(u)=0$ for all $u \in \Omega$ with $u \neq t$. Then $g$ defines an element of $\ell^{1}(\Omega)^{*}$ and we have

$$
\begin{aligned}
0 & =D\left(e_{t} e_{u}\right) \\
& =e_{t} \cdot D\left(e_{u}\right)+D\left(e_{t}\right) \cdot e_{u} \\
& =0+e_{u} D\left(e_{t}\right)-\left\langle D\left(e_{t}\right), e_{u}\right\rangle \phi_{s}
\end{aligned}
$$


for all $t, u \in \Omega$ with $t \neq u$. Now, choose $t, u \in \Omega \backslash\{s\}$ with $t \neq u$, and note that

$$
\left\langle D\left(e_{t}\right), e_{u}\right\rangle=0 \text {. }
$$

A similar argument gives

$$
\begin{aligned}
& \left\langle D\left(e_{s}\right), e_{t}\right\rangle=0 \quad t \in \Omega \\
& \left\langle D\left(e_{t}\right), e_{s}\right\rangle=-\left\langle D\left(e_{t}\right), e_{t}\right\rangle \quad t \in \Omega \backslash\{s\} .
\end{aligned}
$$

For each $a \in \ell^{1}(\Omega)$, note that $a$ can be written as

$$
a=\sum_{t \in c(a)} a(t) e_{t}
$$

where

$$
c(a)=\{t \in \Omega \mid a(t) \neq 0\} .
$$

On the one hand, it follows from (1), (2) and (3)

$$
\begin{aligned}
\langle D(a), b\rangle & =\left\langle D\left(\sum_{u \in c(a)} a(u) e_{u}\right), \sum_{v \in c(b)} b(v) e_{v}\right\rangle \\
& =\sum_{u \in c(a) \cap c(b)} b(u) a(u)\left\langle D\left(e_{u}\right), e_{u}\right\rangle+\sum_{u \in c(a)} b(s) a(u)\left\langle D\left(e_{u}\right), e_{s}\right\rangle \\
& =\sum_{u \in c(a) \cap_{c}(b)} b(u) a(u)\left\langle D\left(e_{u}\right), e_{u}\right\rangle-\sum_{u \in c(a)} b(s) a(u)\left\langle D\left(e_{u}\right), e_{u}\right\rangle
\end{aligned}
$$

for all $a, b \in \ell^{1}(\Omega)$. On the other hand, we have

$$
\begin{aligned}
(a \cdot g-g \cdot a)(b) & =-g(b a)+\phi_{s}(b) g(a) \\
& =-g(b a)+b(s) g(a) \\
& =-\sum_{u \in c(a) \cap c(b)} b(u) a(u) g(u)+\sum_{u \in c(a)} b(s) a(u) g(u) \\
& =\sum_{u \in c(a) \cap c(b)} b(u) a(u)\left\langle D\left(e_{u}\right), e_{u}\right\rangle-\sum_{u \in c(a)} b(s) a(u)\left\langle D\left(e_{u}\right), e_{u}\right\rangle
\end{aligned}
$$

for all $a, b \in \ell^{1}(\Omega)$. Thus $D(a)=a \cdot g-g \cdot a$; that is, $D$ is inner. It follows that

$$
H^{1}\left(\ell^{1}(\Omega), \ell^{1}(\Omega)^{*}\right)=\{0\},
$$

and therefore,

$$
H^{2}\left(\ell^{1}(\Omega),{ }_{0} \mathbb{C}_{\phi_{s}}\right)=\{0\} .
$$

A similar argument shows that $H^{2}\left(\ell^{1}(\Omega),{ }_{0} \mathbb{C}_{0}\right)=\{0\}$.

As a consequence of this result together with Theorem 2.4, we have the following result which shows that the converse of Theorem 2.2 is not valid.

Corollary 2.6. For each set $\Omega$, the Banach algebra $\ell^{1}(\Omega)$ is weakly character amenable.

It is still an open question whether the converse of the implication $(b) \Rightarrow(c)$ in Theorem 2.4 holds.

Question 1. Let $\mathcal{A}$ be a Banach algebra and let $\phi \in \Delta(\mathcal{A}) \cup\{0\}$. Does $H^{2}\left(\mathcal{A},{ }_{0} \mathbb{C}_{\phi}\right)=$ $\{0\}$ when $\mathcal{A}$ is weakly $\phi$-amenable? 


\section{A class of non-weakly character amenable Banach algebras}

In this section, we introduce a class of Banach algebras which are not weakly character amenable. For this purpose, let $\mathcal{A}$ be a Banach algebra, let $\varphi \in \Delta(\mathcal{A}) \cup\{0\}$ and let $\mathcal{Y}$ be a Banach right $\mathcal{A}$-module. Denote by $\mathcal{A} \oplus \oplus_{\varphi} \mathcal{Y}$ the module extension of $\mathcal{A}$ corresponding to ${ }_{\varphi} \mathcal{Y}$; that is, the $\ell^{1}$-direct sum of $\mathcal{A}$ and ${ }_{\varphi} \mathcal{Y}$ as a Banach algebra endowed with the product defined by

$$
(a, y)\left(b, y^{\prime}\right)=\left(a b, \varphi(a) y^{\prime}+y \cdot b\right)
$$

for all $(a, y),\left(b, y^{\prime}\right) \in \mathcal{A} \oplus{ }_{\varphi} \mathcal{Y}$. Then the dual of $\mathcal{A} \oplus \varphi_{\varphi} \mathcal{Y}$ can be identified with the $\ell^{\infty}$-direct sum $\mathcal{A}^{*} \times \mathcal{Y}^{*}$ in the natural way

$$
\langle(f, \Lambda),(a, y)\rangle=f(a)+\Lambda(y)
$$

for all $(f, \Lambda) \in(\mathcal{A} \oplus \varphi \mathcal{Y})^{*}$ and $(a, y) \in \mathcal{A} \oplus \varphi \mathcal{Y}$. Furthermore,

$$
\Delta(\mathcal{A} \oplus \varphi \mathcal{Y})=\{(\psi, 0): \psi \in \Delta(\mathcal{A})\} .
$$

Moreover, the $\left(\mathcal{A} \oplus{ }_{\varphi} \mathcal{Y}\right)$-bimodule actions of $\left.{ }_{\left({ }_{(\varphi, 0)}\right.}\left(\mathcal{A} \oplus{ }_{\varphi} \mathcal{Y}\right)\right)^{*}$ are formulated as follows

$$
(a, y)(f, \Lambda)=(a f+\Lambda(y) \varphi, a \cdot \Lambda)
$$

and

$$
(f, \Lambda) \cdot(a, y)=(\varphi(a) f, \varphi(a) \Lambda)
$$

where $(a, y) \in \mathcal{A} \oplus \varphi \mathcal{Y}$ and $(f, \Lambda) \in \mathcal{A}^{*} \times{ }_{\varphi} \mathcal{Y}^{*}$; see [3] and [29] for more details.

The following result characterizes derivations on $\mathcal{A} \oplus{ }_{\varphi} \mathcal{Y}$.

Theorem 3.1. Let $\mathcal{A}$ be a Banach algebra, let $\varphi \in \Delta(\mathcal{A})$, and let $\mathcal{Y}$ be a Banach right $\mathcal{A}$-module. Then $D: \mathcal{A} \oplus{ }_{\varphi} \mathcal{Y} \longrightarrow\left({ }_{(\varphi, 0)}(\mathcal{A} \oplus \varphi \mathcal{Y})\right)^{*}$ is a derivation if and only if

$$
D(a, y)=\left(D_{\mathcal{A}^{*}}(a)+T(y), D_{\mathcal{Y}^{*}}(a)\right)
$$

for all $(a, y) \in \mathcal{A} \oplus \varphi \mathcal{Y}$ such that

(a) $D_{\mathcal{A}^{*}}: \mathcal{A} \longrightarrow\left({ }_{\varphi} \mathcal{A}\right)^{*}$ is a derivation.

(b) $D_{\mathcal{Y}^{*}}: \mathcal{A} \longrightarrow\left({ }_{\varphi} \mathcal{Y}\right)^{*}$ is a derivation.

(c) $T:{ }_{\varphi} \mathcal{Y} \longrightarrow\left({ }_{\varphi} \mathcal{A}\right)^{*}$ is a bounded linear map with

$$
\begin{gathered}
T(y \cdot a)=D_{\mathcal{Y}^{*}}(a)(y) \varphi+\varphi(a) T(y), \\
\varphi(a) T(y)=a T(y) .
\end{gathered}
$$

for all $a \in \mathcal{A}$ and $y \in \mathcal{Y}$. In this case,

(i) $D$ is inner if and only if $D_{\mathcal{A}^{*}}$ is inner and there is $\Lambda \in \mathcal{Y}^{*}$ such that $T(y)=$ $\Lambda(y) \varphi$ for all $y \in \mathcal{Y}$.

(ii) If there is $\Lambda \in \mathcal{Y}^{*}$ such that $T(y)=\Lambda(y) \varphi$, then $D_{\mathcal{Y}^{*}}=a d_{\Lambda}$.

Proof. Suppose that $D: \mathcal{A} \oplus{ }_{\varphi} \mathcal{Y} \longrightarrow\left({ }_{(\varphi, 0)} \mathcal{A} \oplus{ }_{\varphi} \mathcal{Y}\right)^{*}$ is a derivation. Then

$$
D(a, y)=D(a, 0)+D(0, y)
$$


for all $a \in \mathcal{A}$ and $y \in \mathcal{Y}$. Since $D(a, 0), D(0, y) \in \mathcal{A}^{*} \times \mathcal{Y}^{*}$, we can define the maps

$$
\begin{gathered}
D_{\mathcal{A}^{*}}: \mathcal{A} \longrightarrow\left({ }_{\varphi} \mathcal{A}\right)^{*}, \\
D_{\mathcal{Y}^{*}}: \mathcal{A} \longrightarrow\left({ }_{\varphi} \mathcal{Y}\right)^{*}, \\
T:{ }_{\varphi} \mathcal{Y} \longrightarrow\left({ }_{\varphi} \mathcal{A}\right)^{*},
\end{gathered}
$$

and

$$
S:{ }_{\varphi} \mathcal{Y} \longrightarrow\left({ }_{\varphi} \mathcal{Y}\right)^{*}
$$

such that

$$
D(a, 0)=\left(D_{\mathcal{A}^{*}}(a), D_{\mathcal{Y}^{*}}(a)\right),
$$

and

$$
D(0, y)=(T(y), S(y))
$$

Then

$$
D(a, y)=\left(D_{\mathcal{A}^{*}}(a)+T(y), D_{\mathcal{Y}^{*}}(a)+S(y)\right) .
$$

For every $(a, y),\left(a^{\prime}, y^{\prime}\right) \in \mathcal{A} \oplus{ }_{\varphi} \mathcal{Y}$, on the one hand,

$$
\begin{aligned}
D\left((a, y)\left(a^{\prime}, y^{\prime}\right)\right) & =D\left(\left(a a^{\prime}, \varphi(a) y^{\prime}+y \cdot a^{\prime}\right)\right) \\
& =\left(D_{\mathcal{A}^{*}}\left(a a^{\prime}\right)+T\left(\varphi(a) y^{\prime}+y \cdot a^{\prime}\right), D \mathcal{Y}^{*}\left(a a^{\prime}\right)+S\left(\varphi(a) y^{\prime}+y \cdot a^{\prime}\right)\right),
\end{aligned}
$$

and on the other hand

$$
\begin{array}{r}
(a, y)\left(D_{\mathcal{A}^{*}}\left(a^{\prime}\right)+T\left(y^{\prime}\right), D_{\mathcal{Y}^{*}}\left(a^{\prime}\right)+S\left(y^{\prime}\right)\right)+\varphi\left(a^{\prime}\right)\left(\left(D_{\mathcal{A}^{*}}(a)+T(y), D_{\mathcal{Y}^{*}}(a)+S(y)\right)\right. \\
=\left(a D_{\mathcal{A}^{*}}\left(a^{\prime}\right)+a T\left(y^{\prime}\right)+D_{\mathcal{Y}^{*}}\left(a^{\prime}\right)(y) \varphi+S\left(y^{\prime}\right)(y) \varphi, a \cdot D_{\mathcal{Y}^{*}}\left(a^{\prime}\right)+a \cdot S\left(y^{\prime}\right)\right) \\
+\left(\varphi\left(a^{\prime}\right) D_{\mathcal{A}^{*}}(a)+\varphi\left(a^{\prime}\right) T(y), \varphi\left(a^{\prime}\right) D_{\mathcal{Y}^{*}}(a)+\varphi\left(a^{\prime}\right) S\left(y^{\prime}\right)\right)
\end{array}
$$

Since $D$ is a derivation,

$$
\begin{aligned}
D_{\mathcal{A}^{*}}\left(a a^{\prime}\right)+T\left(\varphi(a) y^{\prime}+y \cdot a^{\prime}\right) & =a D_{\mathcal{A}^{*}}\left(a^{\prime}\right)+a T\left(y^{\prime}\right)+D_{\mathcal{Y}^{*}}\left(a^{\prime}\right)(y) \varphi+S\left(y^{\prime}\right)(y) \varphi \\
& +\varphi\left(a^{\prime}\right) D_{\mathcal{A}^{*}}(a)+\varphi\left(a^{\prime}\right) T(y)
\end{aligned}
$$

and

$D_{\mathcal{Y}^{*}}\left(a a^{\prime}\right)+S\left(\varphi(a) y^{\prime}+y \cdot a^{\prime}\right)=a \cdot D_{\mathcal{Y}^{*}}\left(a^{\prime}\right)+a \cdot S\left(y^{\prime}\right)+\varphi\left(a^{\prime}\right) D_{\mathcal{Y}^{*}}(a)+\varphi\left(a^{\prime}\right) S\left(y^{\prime}\right)$

If we take $y=y^{\prime}=0$, it follows that

$$
D_{\mathcal{A}^{*}}\left(a a^{\prime}\right)=a D_{\mathcal{A}^{*}}\left(a^{\prime}\right)+\varphi\left(a^{\prime}\right) D_{\mathcal{A}^{*}}(a),
$$

and

$$
D_{\mathcal{Y}^{*}}\left(a a^{\prime}\right)=a \cdot D_{\mathcal{Y}^{*}}\left(a^{\prime}\right)+\varphi\left(a^{\prime}\right) D_{\mathcal{Y}^{*}}(a) .
$$

If we take $a=a^{\prime}=0$, it follows that $S\left(y^{\prime}\right)(y)=0$ for all $y, y^{\prime} \in \mathcal{Y}$, and hence $S=0$. If we take $a=y^{\prime}=0$, it follows that

$$
T\left(y \cdot a^{\prime}\right)=D_{\mathcal{X} *}\left(a^{\prime}\right)(y) \varphi+\varphi\left(a^{\prime}\right) T(y),
$$


and if we take $a^{\prime}=y=0$, it follows that

$$
T\left(a \cdot y^{\prime}\right)=T\left(\varphi(a) y^{\prime}\right)=a T\left(y^{\prime}\right)
$$

The converse is an easy computation.

To prove (i), in this case suppose that $D$ is an inner derivation. Then there is $(f, \Lambda) \in(\mathcal{A} \oplus \varphi \mathcal{Y})^{*}$ such that

$$
D(a, y)=(a, y)(f, \Lambda)-\varphi(a)(f, \Lambda)
$$

Therefore,

$$
D_{\mathcal{A}^{*}}(a)+T(y)=a f+\Lambda(y) \varphi-\varphi(a) f
$$

and

$$
D_{\mathcal{Y} *}(a)=a \cdot \Lambda-\varphi(a) \Lambda
$$

That is, $D_{\mathcal{Y}^{*}}=\operatorname{ad}_{\Lambda}, T(y)=\Lambda(y) \varphi$ and

$$
D_{\mathcal{A}^{*}}(a)=a f-\varphi(a) f .
$$

The converse of (i) and the statement (ii) are obvious.

As a consequence of Theorem 3.1, we have the following result. First, for a Banach space $\mathcal{E}$, we denote by ${ }_{\varphi} \mathcal{E}_{\varphi}$ the Banach $\mathcal{A}$-bimodule obtained from $\mathcal{E}$ by putting

$$
a \cdot \xi:=\varphi(a) \xi:=\xi \cdot a
$$

for all $a \in \mathcal{A}$ and $\xi \in \mathcal{E}$.

Corollary 3.2. Let $\mathcal{A}$ be a Banach algebra, let $\varphi \in \Delta(\mathcal{A})$, and let $\mathcal{E}$ be a Banach space. Then $D: \mathcal{A} \oplus_{\varphi} \mathcal{E}_{\varphi} \longrightarrow\left({ }_{(\varphi, 0)}\left(\mathcal{A} \oplus_{\varphi} \mathcal{E}_{\varphi}\right)\right)^{*}$ is a derivation if and only if

$$
D(a, \xi)=\left(D_{\mathcal{A}^{*}}(a)+T(\xi), 0\right)
$$

for all $a \in \mathcal{A}$ and $\xi \in \mathcal{E}$ such that

(a) $D_{\mathcal{A}^{*}}: \mathcal{A} \longrightarrow\left({ }_{\varphi} \mathcal{A}\right)^{*}$ is a derivation.

(b) $T:{ }_{\varphi} \mathcal{E}_{\varphi} \longrightarrow\left({ }_{\varphi} \mathcal{A}\right)^{*}$ is a bounded linear map such that for each $a \in \mathcal{A}$ and $\xi \in \mathcal{E}$,

$$
\varphi(a) T(\xi)=a T(\xi)
$$

In this case, $D$ is inner if and only if $D_{\mathcal{A}^{*}}$ is inner and there is $\Lambda \in \mathcal{E}^{*}$ such that $T(\xi)=\Lambda(\xi) \varphi$ for all $\xi \in \mathcal{E}$.

Let $\mathcal{E}$ be a Banach space and let $\alpha \in \mathcal{E}^{*} \backslash\{0\}$. Then $\mathcal{E}$ with the multiplication $\xi \xi^{\prime}=\alpha\left(\xi^{\prime}\right) \xi$ for all $\xi^{\prime} \xi^{\prime} \in \mathcal{E}$ is a Banach algebra which is denoted by $\operatorname{Alg}_{\alpha}(\mathcal{E})$. It is easy to see that

$$
\Delta\left(\operatorname{Alg}_{\alpha}(\mathcal{E})\right)=\{\alpha\} .
$$

Proposition 3.3. Suppose that $\mathcal{E}$ is a Banach space with $\operatorname{dim}(\mathcal{E}) \geq 2$. Then $A \lg _{\alpha}(\mathcal{E}) \oplus$ ${ }_{\alpha}\left(\operatorname{Alg}_{\alpha}(\mathcal{E})^{*}\right)_{\alpha}$ is not weakly $(\alpha, 0)$-amenable. 
Proof. Suppose to the contrary that $\operatorname{Alg}_{\alpha}(\mathcal{E}) \oplus{ }_{\alpha}\left(\operatorname{Alg}_{\alpha}(\mathcal{E})^{*}\right)_{\alpha}$ is weakly $(\alpha, 0)$-amenable. Let $I: \operatorname{Alg}_{\alpha}(\mathcal{E})^{*} \longrightarrow \operatorname{Alg}_{\alpha}(\mathcal{E})^{*}$ be the identity mapping and define

$$
D: \operatorname{Alg}_{\alpha}(\mathcal{E}) \oplus_{\alpha}\left(\operatorname{Alg}_{\alpha}(\mathcal{E})^{*}\right)_{\alpha} \longrightarrow\left({ }_{(\alpha, 0)}\left(\operatorname{Alg}_{\alpha}(\mathcal{E}) \oplus_{\alpha}\left(\operatorname{Alg}_{\alpha}(\mathcal{E})^{*}\right)_{\alpha}\right)\right)^{*}
$$

given by

$$
D(\xi, f):=(I(f), 0)
$$

for all $\xi \in \operatorname{Alg}_{\alpha}(\mathcal{E})$ and $f \in \operatorname{Alg}_{\alpha}(\mathcal{E})^{*}$. Then $D$ is a derivation. By Corollary 3.2, there is $m_{0} \in \operatorname{Alg}_{\alpha}(\mathcal{E})^{* *}$ such that $f=m_{0}(f) \alpha$ for all $f \in \operatorname{Alg}_{\alpha}(\mathcal{E})^{*}$. It follows that $\mathcal{E}^{*}$ is generated by $\{\alpha\}$ which is a contradiction.

Theorem 3.4. Let $\mathcal{A}$ be a Banach algebra and let $\mathcal{Y}$ be a Banach right $\mathcal{A}$-module. Then $D: \mathcal{A} \oplus_{0} \mathcal{Y} \longrightarrow\left({ }_{(0,0)}\left(\mathcal{A} \oplus_{0} \mathcal{Y}\right)\right)^{*}$ is a derivation if and only if

$$
D(a, y)=\left(D_{\mathcal{A}^{*}}(a)+T(y), D_{\mathcal{Y}^{*}}(a)+S(y)\right)
$$

for all $a \in \mathcal{A}$ and $y \in \mathcal{Y}$ such that

(a) $D_{\mathcal{A}^{*}}: \mathcal{A} \longrightarrow\left({ }_{0} \mathcal{A}\right)^{*}$ is a derivation.

(b) $D_{\mathcal{Y}^{*}}: \mathcal{A} \longrightarrow\left({ }_{0} \mathcal{Y}\right)^{*}$ is a derivation.

(c) $T:{ }_{0} \mathcal{Y} \longrightarrow\left({ }_{0} \mathcal{A}\right)^{*}$ is an $\mathcal{A}$-bimodule homomorphism.

(d) $S:{ }_{0} \mathcal{Y} \longrightarrow(0 \mathcal{Y})^{*}$ is an $\mathcal{A}$-bimodule homomorphism.

In this case, $D$ is inner if and only if $D_{\mathcal{A}^{*}}$ and $D_{\mathcal{Y}^{*}}$ are inner, $T=0$ and $S=0$.

Proof. The proof is similar to the proof of Theorem 3.1.

At the end of this section, we start making a comparison between some different versions of amenability related to weak character amenability.

Remark 3.5. (a) Character amenability implies automatically weak character amenability. The converse of this statement is false. For an example, let $G$ be a locally compact group and let $M(G)$ be the measure algebra of $G$. Since $M(G)$ has an identity, it follows that $M(G)$ is weakly character amenable by Theorem 2.2. Moreover, it is known that $M(G)$ is weakly amenable if and only if $G$ is discrete; see Theorem 1.2 of [4]. Also, $M(G)$ is character amenable if and only if $G$ is discrete and amenable; see Corollary 2.5 of [22]. So, in the case where $G$ is non-discrete, $M(G)$ is weakly character amenable, but neither weakly amenable nor character amenable.

(b) Let us remark that amenability implies character amenability and weak amenability. But the converse is not true. For example, consider the $C^{*}$-algebra $B(H)$ of all bounded operators on an infinite-dimensional Hilbert space $H$. In fact, every $C^{*}$-algebra is both weakly amenable and character amenable; see [9] and [12]. Moreover, a $C^{*}$-algebra is amenable if and only if it is nuclear. So, we only need to recall from [8] that $B(H)$ is not nuclear.

(c) Let $\mathbb{N}$ denote the natural number and consider the Banach algebra $\ell^{2}(\mathbb{N})$ of all sequences $a:=(a(n))$ of complex numbers with

$$
\|a\|_{2}:=\sum_{n=1}^{\infty}|a(n)|^{2}<\infty
$$


endowed with the pointwise product. Then $\ell^{2}(\mathbb{N})$ is weakly amenable by Example 4.1 .42 (iii) [3], whereas in Example 2.3, we have shown that $\ell^{2}(\mathbb{N})$ is not weakly character amenable.

We complete the following schematic diagram that illustrates the implications between weak character amenability and some related notions of amenability. Here "-" indicates the fact that there is a counter-example to the relevant implication.

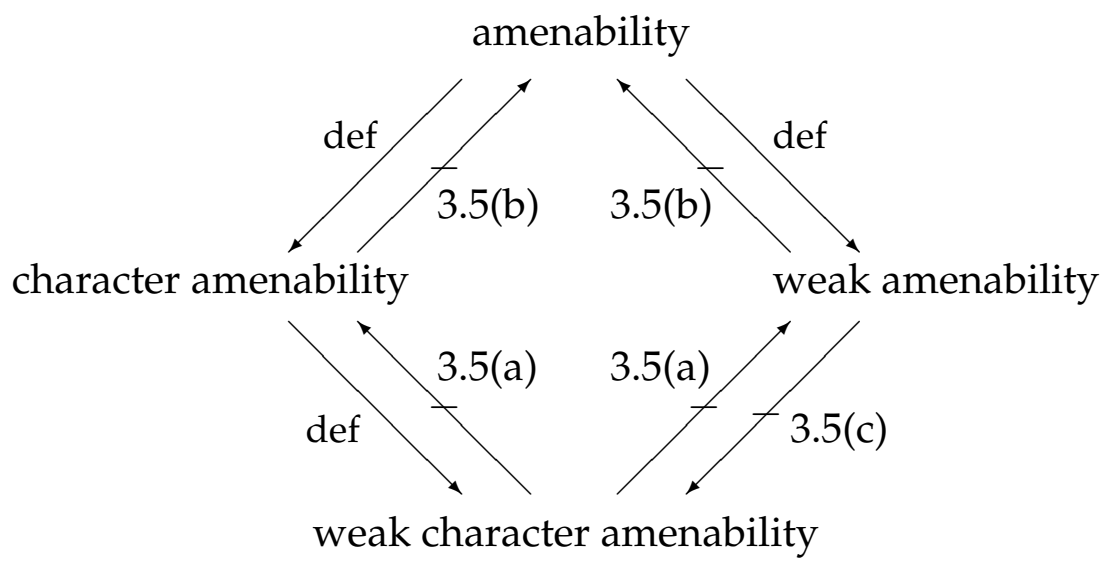

Acknowledgements. The authors would like to sincerely thank the referee for invaluable his/her suggestions and comments on the paper. They acknowledge that the research was supported by a grant from INSF, Iran National Science Foundation, under the name Fourier Analysis and Applications.

\section{References}

[1] F. AKHTARI AND R. NASR-ISFAHANI, Strongly amenable representations of involutive Banach algebras, Positivity 18 (2014), 395-412.

[2] X. Chen, A. T. LAU, AND C. K. NG, A property for locally convex *-algebras related to property (T) and character amenability, Studia Math. 227 (2015), 259-286.

[3] H. G. DALES, Banach algebras and Automatic Continuity, Clarendon Press. Oxford, (2000).

[4] H. G. Dales, F. Ghahramani, And A. Ya. Helemskil, The amenability of measure algebras, J. London Math. Soc. 66 (2002), 213-226.

[5] M. DAshti, R. NAsR-Isfahani, AND S. SOltani RenAni, Character amenability of Lipschitz algebras, Canad. Math. Bull. 57 (2014), no. 1, 37-41.

[6] S. Desaulniers, R. NAsR-Isfahani, AND M.Nemati, Common fixed point properties and amenability of a class of Banach algebras, J. Math. Anal. Appl. 402 (2011), 225-232. 
[7] G. H. Esslamzadeh And B. ShojaeE, Approximate weak amenability of Banach algebras, Bull. Belg. Math. Soc. 18 (2011), no. 3, 415-429.

[8] U. HaAgeruP, All nuclear $C^{*}$-algebras are amenable, Invent. Math. 74 (1983), 305-319.

[9] U. HAAGERUP AND N. J. LAUSTSEN, Weak amenability of $C^{*}$-algebras and a theorem of Goldstein, In Banach algebras. 97 (ed. E. Albrecht and M. Mathieu), Walter de Gruyter, Berlin, (1998), 223-243.

[10] B. E. Johnson, Cohomology in Banach algebras, Mem. Amer. Math. Soc. 127, (1972).

[11] B. E. JoHnson, Derivations from $L^{1}(G)$ into $L^{1}(G)$ and $L^{\infty}(G)$, Harmonic analysis (Luxembourg, 1987), 191-198, Lecture Notes in Math, 1359, Springer-Verlag, Berlin, (1988).

[12] E. KANIUTH, A. T. LAU, AND J. PYM, On $\phi$-amenability of Banach algebras, Math. Proc. Camb. Phil. Soc. 144 (2008), 85-96.

[13] E. KANIUTH, A. T. LAU, AND J. PyM, On character amenability of Banach algebras, J. Math. Anal. Appl. 344 (2008), 942-955.

[14] M. LASHKARIZAde BAmi AND H. SAmEA, Amenability and essential amenability of certain convolution Banach algebras on compact hypergroups, Bull. Belg. Math. Soc. 16, (2009), no. 1, 145-152.

[15] A. T. LAU, Invariant means on subsemigroups of locally compact groups, Rocky Mountain J. Math. 3 (1973), 77-81.

[16] A. T. LAU, Analysis on a class of Banach algebras with application to harmonic analysis on locally compact groups and semigroups, Fund. Math. 118 (1983), 161-175.

[17] A. T. LAU, Uniformly continuous functionals on Banach algebras, Colloq. Math. 51 (1987), 195-205.

[18] A. T. LAU AND R. J. LOY, Weak amenability of Banach algebras on locally compact groups, J. Funct. Anal. 145 (1997), no. 1, 175-204.

[19] A. T. LAU AND J. LUDWIG, Fourier-Stieltjes algebra of a topological group, Adv. Math. 229 (2012), 2000-2023.

[20] A. T. LAU AND J. C. S. WONG, Invariant subspaces for algebras of linear operators and amenable locally compact groups, Proc. Amer. Math. Soc. 102 (1988), 581-586.

[21] B. MOHAmmadzadeh And R. NAsR-Isfahani, Positive elements of left amenable Lau algebras, Bull. Belg. Math. Soc. 13 (2006), no. 2, 319-324.

[22] M. S. Monfared, Character amenability of Banach algebras, Math. Proc. Camb. Phil. Soc. 144 (2008), 697-706. 
[23] R. NASR-Isfahani AND S. SOltani RenANI, Character contractibility of Banach algebras and homological properties of Banach modules, Studia Math. 202 (2011), 205-225.

[24] R. NASR-ISFAHANI AND S. SOLTANI RENANI, Character injectivity and projectivity of Banach modules, Quart. J. Math. Oxford 65 (2014), no. 2, 665-676.

[25] M. NemATI, On $\phi$-ergodic property of Banach modules, Bull. Belg. Math. Soc. $22(2015)$.

[26] A. SAhami And A. Pourabbas, On $\phi$-biflat and $\phi$-biprojective Banach algebras, Bull. Belg. Math. Soc. 20 (2013), no. 5, 789-801.

[27] J. P. PIER, Amenable Banach Algebras, Pitman research notes in mathematics series, Vol. 172, Longman scientific and technical, Harlow, (1988).

[28] V. RundE, Lectures on Amenability, Springer-Verlag, New York, (2002).

[29] Y. ZHANG, Weak amenability of module extensions of Banach algebras, Trans. Amer. Math. Soc. 354 (2002), 4131-4151.

Department of Mathematical Sciences, Isfahan University of Technology,

Isfahan 84156-83111, Iran

emails: isfahani@cc.iut.ac.ir, shahmoradi@math.iut.ac.ir, simasoltani@cc.iut.ac.ir 